\title{
Administration of Vitamin D3 Improves Hemoglobin Level by Regulating TNF- $\alpha$ and IL-6 in DSS-induced Colitis Mice
}

\author{
Ervin Monica $^{1, *}$, Primayuni Dhia Hasanah ${ }^{1}$, Arief Fadillah ${ }^{1}$, Rara Aulia $^{1}$, \\ Eko Sulistijono $^{2}$, Satrio Wibowo ${ }^{3}$
}

\begin{abstract}
${ }^{1}$ Master Program of Biomedical Science, Graduate Program, Faculty of Medicine, Universitas Brawijaya, Jl. Veteran, Malang, Indonesia ${ }^{2}$ Department of Pediactrics, Division of Neonatology, Saiful Anwar General Hospital, Universitas Brawijaya, Jl. Veteran, Malang, Indonesia ${ }^{3}$ Department of Pediatrics, Division of Pediatric Gastroenterology and Hepatology, Saiful Anwar General Hospital, Universitas Brawijaya, Jl. Veteran, Malang, Indonesia

*Corresponding author. E-mail: ervinevin@gmail.com
\end{abstract}

Received date: Nov 17, 2019; Revised date: Mar 23, 2020; Accepted date: Mar 26, 2020

\section{Abstract}

ACKGROUND: Anemia is frequently found in ulcerative colitis (UC) patients and assumed to be related to inflammatory process. Vitamin D3 (VD) is known to have anti-inflammatory and immunomodulatory effects. It also has the potential to be an alternative treatment of the inflammatory process that occurs at UC, however its mechanism has not been clearly established. This study aimed to assess the effect of VD on histopathology and hemoglobin levels in UC through its regulation in tumor necrosis factor (TNF)- $\alpha$ and interleukin (IL)-6.

METHODS: Total samples of 24 mice were divided equally into Sham group, UC group, UC+VD group (given $3 \%$ dextran sodium sulfate (DSS) followed by VD), and VD+UC group (given VD followed by $3 \%$ DSS). Mouse Colitis Histology Index (MCHI) was used to measure histopathological changes. Immunohistochemical staining was used to observe expression of TNF- $\alpha$ and IL-6 in colon. Evaluation of anemia was determined by hemoglobin levels.

\section{Introduction}

Ulcerative colitis (UC) is characterized by diffuse inflammation of the colon and rectum mucosa.(1) Based on colonoscopy data from Saiful Anwar General Hospital in 2010-2014, 176 patients were diagnosed with UC or $8.2 \%$ of the total colonoscopy patients.(2) A combination of genetic, environmental, and microbial factors causes
RESULTS: Based on MCHI scores, significant epithelial damage was found in colon sample of UC group $(8.25 \pm 3.05)$ compared to Sham $(0.33 \pm 0.26), \mathrm{UC}+\mathrm{VD}(2.33 \pm 1.07)$, and VD+UC group $(2.83 \pm 0.75) \quad(p<0.05)$. Significant lower numbers of TNF- $\alpha$ were found in Sham (27.33 \pm 3.42$)$, $\mathrm{UC}+\mathrm{VD}(36.33 \pm 1.86)$, and VD+UC group $(36.68 \pm 1.86)$ compared with UC group (44.66 \pm 4.87$)(p<0.05)$. Significant less IL-6 expression was found in Sham (18.05 \pm 2.96$)$, $\mathrm{UC}+\mathrm{VD}(24.78 \pm 0.79)$, and VD+UC group (25.09 \pm 2.79$)$ compared to UC group $(38.85 \pm 3.51)(p<0.05)$. Differences in hemoglobin levels were significantly lower in UC group (11.85 \pm 0.97$)$ compared to Sham (14.25 \pm 0.47$)$, UC+VD (13.68 \pm 0.68$)$, VD+UC group (13.52 \pm 1.07$)(p<0.05)$.

CONCLUSION: VD significantly reduced proinflammatory cytokines, increased mucosal repair, and improved hemoglobin levels.

KEYWORDS: colitis, ulcerative, interleukin-6, tumor necrosis factor-alpha

Indones Biomed J. 2020; 12(2): 130-5 colitis by disrupting immune system regulation in the form of changes in the balance of T helper cells to Th1 and Th17 compared to Th2.(3) If not treated properly, colitis can cause various complications such as anemia, as one of the most common extraintestinal complications.(4) The discharge of blood from the digestive tract continuously resulting in iron deficiency is what usually causes anemia in colitis.(5) Increased activity of pro-inflammatory cytokines such as tumor necrosis factor (TNF)- $\alpha$ and 
interleukin (IL)-6 also can cause anemia in colitis. TNF- $\alpha$ increases ferritin production which reduces the amount of iron for erythropoiesis, and IL-6 initiates the formation of hepcidin as a negative regulator of iron absorption and iron release. These mechanisms disrupt the erythropoietin (EPO) signaling process and reduce the regulation of EPO receptors. Thereby EPO resistance is induced in erythroid progenitor cells. $(5,6)$ Several studies have found low levels of vitamin D in colitis patients. Vitamin D deficiency plays a critical role in the severity of colitis by causing an increase in pro-inflammatory cytokines, decreased expression of tight junction, and bacterial clearance of intestinal epithelium. $(7,8)$

Vitamin D refers to a group of fat-soluble secosteroids, and the primary compounds are vitamin D3 (cholecalciferol) and vitamin D2 (ergocalciferol). The biggest abundant source of vitamin D in our body is cholecalciferol synthesis in the skin through direct sun exposure. Ultraviolet-B (UVB) from the sun is absorbed by the skin that activates the synthesis of vitamin D.(9) The active form of vitamin D, vitamin D3 or calcitriol is known to take part in regulating various inflammatory cytokines so that an equilibrium can be achieved between pro-inflammatory cytokines (TNF- $\alpha$, IL-6) and anti-inflammatory cytokines.(10-13) Then, with the controlled expression of various inflammatory cytokines, hepcidin formation can be inhibited to prevent the occurrence of anemia.(14)

\section{Methods}

The mice used in this study was obtained from Pusvetma, Surabaya, Indonesia. Treatment of animal models, immunohistochemistry (IHC) staining for TNF- $\alpha$ and IL6, hematoxylin-eosin (H\&E) staining for measurement of epithelial damage, and measurement of hemoglobin level were all conducted at Faculty of Medicine, Universitas Brawijaya. Ethical clearance was obtained from the Ethics Committee of Faculty of Medicine, Brawijaya University, with number: 434/EC/KEPK/12/2017.

\section{Treatment of Mice}

This study used twenty-four 8 weeks old male Balb/c mice weighing 17-25 grams. The mice were randomly divided into 4 groups after 1 week of acclimatization. Distilled water was given to the sham group for 14 days. Colitis group was given 3\% of dextran sodium sulfate (DSS) (Cat No. ab146569, Abcam, Cambridge, UK) for 7 days and followed by distilled water for 7 days. The first treatment group
(UC+VD) was given $3 \%$ of DSS for 7 days and followed by vitamin D3 (Oscal, Calcitriol, Dankos Farma, Jakarta, Indonesia) at a dose of $0.2 \mathrm{ug} / 25 \mathrm{~g} /$ day via intragastric for 7 days. The second treatment group (VD+UC) was given vitamin D3 at a dose of $0.2 \mathrm{ug} / 25 \mathrm{~g} /$ day via intragastric for 7 days and followed by $3 \%$ of DSS for 7 days. After DSS induction and vitamin D3 administration in the treatment group were completed, all mice were dissected to collect blood samples and colonic tissue. The colitis condition was confirmed by the epithelial damage in histopathological colon after dissection. The distal colon was taken to make paraffin block and sliced using a microtome. Last, H\&E and IHC staining were observed using the blind method.

\section{Histopathological Analysis}

A 5-mm distal colonic portion was formed in paraffin and H\&E stain. The Mouse Colitis Histology Index (MCHI) score was used to measure colonic epithelial damage. MCHI $=(1 \mathrm{x}$ Goblet cells disappear $)+(2 \mathrm{x}$ crypt density $)+$ ( $2 \mathrm{x}$ crypt hyperplasia $)+(3 \mathrm{x}$ submucosal infiltration $) .(15)$

\section{Hemoglobin Analysis}

Blood samples were taken and were diluted in the WBC chamber then measured by Spectrophotometry, through optical pathways in the white blood cell (WBC) room. Then the reading was carried out at $\lambda 532 \mathrm{~nm}$ with a spectrophotometer using the Hematology Analyzer ABX Micro 60 (Horiba ABX SAS, Irvine, California, USA), which can be used to analyze hemoglobin levels in mice. Hemoglobin levels were expressed in grams per deciliter $(\mathrm{g} / \mathrm{dL})$

\section{IHC Staining of TNF Alpha and IL-6 Expression}

Colon parts were fixed in neutral buffer formalin and embedded in paraffin. Then, they were deparaffinized, as well as sequentially rehydrated using xylol, absolute ethanol, $90 \%$ ethanol, $80 \%$ ethanol, $70 \%$ ethanol, sterile distillated aqua, $3 \% \mathrm{H}_{2} \mathrm{O}_{2}$, and blocking buffer. The parts were examined with Rabbit polyclonal TNF- $\alpha$ (Cat No. bs2081R, Bioss, Woburn, USA) and IL-6 (Cat No. bs-0782R, Bioss, Woburn, USA), then incubated overnight at $4^{\circ} \mathrm{C}$. After incubation, they were thoroughly washed with phosphate buffer saline (PBS) for 5 minutes and repeated three times. Subsequently, the colon parts were incubated with secondary antibody at room temperature for 60 minutes, then with horseradish peroxidase at room temperature for 40 minutes. Reaction products were visualized by diaminobenzidine (Nichirei Bioscience Inc, Tokyo, Japan) and hematoxylin. Then, cells expressing TNF- $\alpha$ and IL- 6 in the form of 
cells with a flat, brownish core were observed under the microscope in 20 fields of view with 400x magnification. Last, the mean scores were collected.

\section{Statistical Analysis}

Data were expressed as mean \pm standard error of the mean (SEM). Comparisons between the 4 groups (Sham, UC, $\mathrm{UC}+\mathrm{VD}$, and $\mathrm{VD}+\mathrm{UC}$ ) were analyzed using analysis of variance (ANOVA) test. A comparison between two different groups was carried out using the Post Hoc LSD test. Kruskal-Wallis and Post Hoc Mann-Whitney tests were used for abnormal and non-homogeneous data. The $p$-values $<0.05$ were considered statistically significant. All statistical analyses were conducted using IBM SPSS software ver. 23.0 (IBM Co., Armonk, NY, USA).

\section{Results}

\section{Vitamin D3 and Reduction of Epithelial Damage}

The mice model of colitis was induced by $3 \%$ of DSS through drinking water. Epithelial damage increased in the UC group compared to other groups (Figure 1). In Figure 1, two observers found significant epithelial damage in colon samples of UC group, marked by the loss of goblet cells, decreased cryptic density, hyperplasia, and leukocytes infiltration into sub-mucosa in the UC colon. Comparisons of MCHI scores of the samples showed significant increases in UC $(8.25 \pm 3.05)$ compared to sham $(0.33 \pm 0.26)$, UC compared to UC+VD $(2.33 \pm 1.07)$, and UC compared to VD+UC $(2.83 \pm 0.75) \quad(p<0.05)$. However, there was no significant difference found between Sham, UC+VD and $\mathrm{VD}+\mathrm{UC}$ groups $(p>0.05)$.

\section{Hemoglobin Level}

This research evaluated colitis-induced anemia to determine the potential of vitamin D3 in effectively increasing hemoglobin levels. The mean hemoglobin levels of mice after the treatment were measured $(\mathrm{g} / \mathrm{dL})$ (Table 1). The levels of hemoglobin in UC group (11.85 \pm 0.97$)$ decreased compared to other groups. ANOVA test results showed significant differences between each treatment in the parameter measured at $\alpha=0.05$. Table 2 showed that $U C+V D$ group (13.68 \pm 0.68$)$ and VD+UC group (13.52 \pm 1.07$)$ had a significant increase in hemoglobin effect at a $95 \%$ confidence level compared to UC group. There was no significant difference found between Sham, UC+VD and $\mathrm{VD}+\mathrm{UC}$ groups $(p>0.05)$.
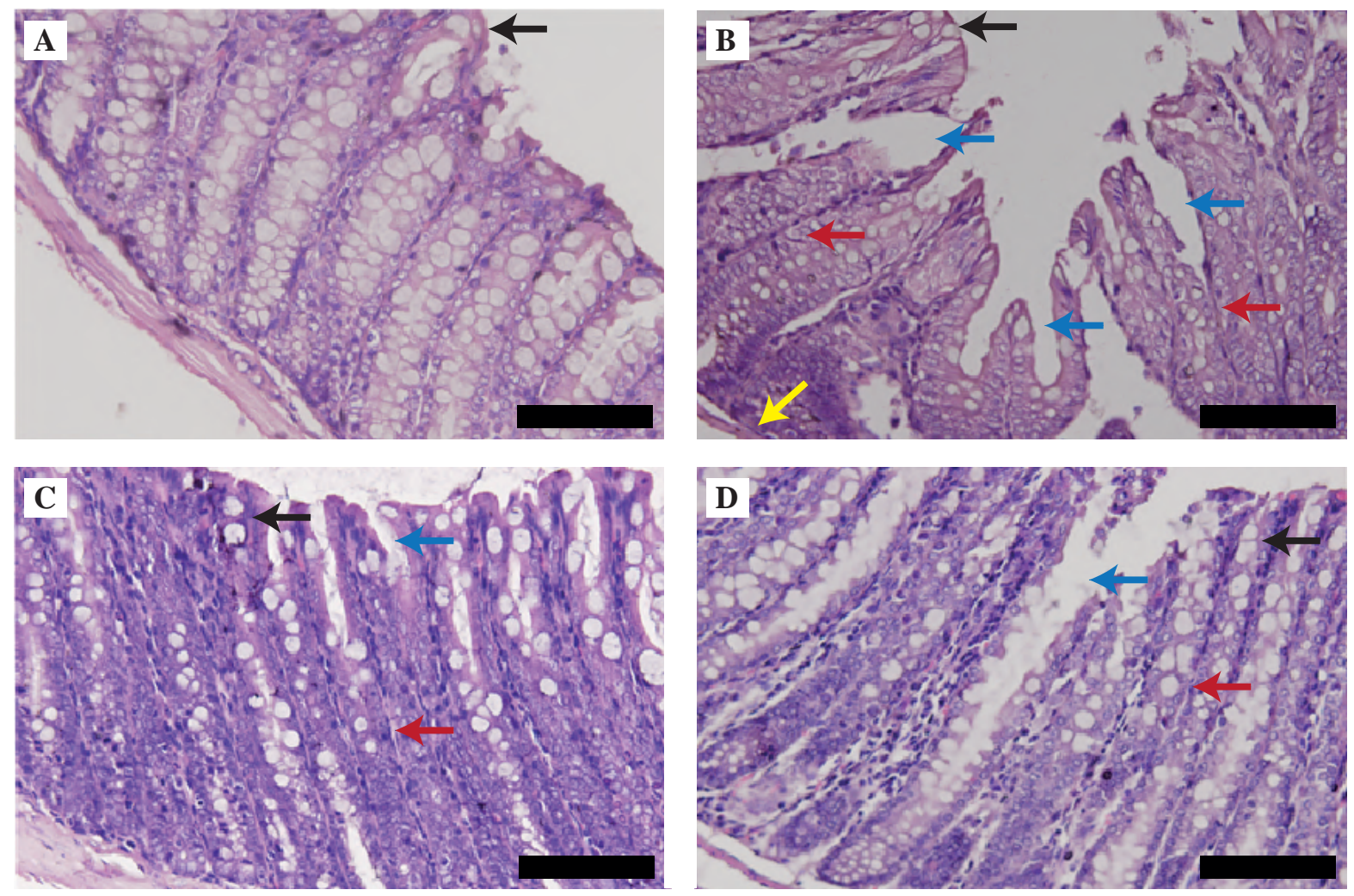

Figure 1. Light microscopy observation of the colon tissue samples of mice following haematoxylin and eosin staining. Black arrow: goblet cell; Blue arrow: decreased crypte density; Red arrow: hyperplasia crypte; Yellow arrow: leukocytes infiltration. Black bar: 100 $\mu$ m. 
Table 1. Multiple comparisons of hemoglobin levels.

\begin{tabular}{|c|c|c|c|c|c|}
\hline \multirow{2}{*}{ Groups } & \multicolumn{4}{|c|}{ Post Hoc LSD } & \multirow{2}{*}{$p$-value } \\
\hline & Sham & $\mathbf{U C}$ & $\mathrm{UC}+\mathrm{VD}$ & VD+UC & \\
\hline Sham (14.25 \pm 0.47$)$ & - & $0.000^{*}$ & 0.252 & 0.143 & 0.000 \\
\hline UC (11.85 \pm 0.97$)$ & $0.000 *$ & - & $0.001 *$ & $0.002 *$ & \\
\hline $\mathrm{UC}+\mathrm{VD}(13.68 \pm 0.68)$ & 0.252 & $0.001 *$ & - & 0.732 & \\
\hline VD+UC (13.52 \pm 1.07$)$ & 0.143 & $0.002 *$ & 0.732 & - & \\
\hline
\end{tabular}

"Significancy was tested with ANOVA $(p<0.05)$.

\section{Vitamin D3 and TNF- $\alpha$ Expression}

TNF- $\alpha$ (cell/field of view) expression of colonic samples after IHC staining (Figure 2) in Sham (27.33 \pm 3.42 ), UC+VD (36.33 \pm 1.86$)$, and $\mathrm{VD}+\mathrm{UC}(38.68 \pm 3.68)$ groups showed a significant smaller number of TNF- $\alpha$ cells compared to UC (44.66 \pm 4.87$)(p<0.05)$. Meanwhile, there were no significant differences between the $\mathrm{UC}+\mathrm{VD}$ and $\mathrm{VD}+\mathrm{UC}$ groups ( $p>0.05)$ (Table 2).
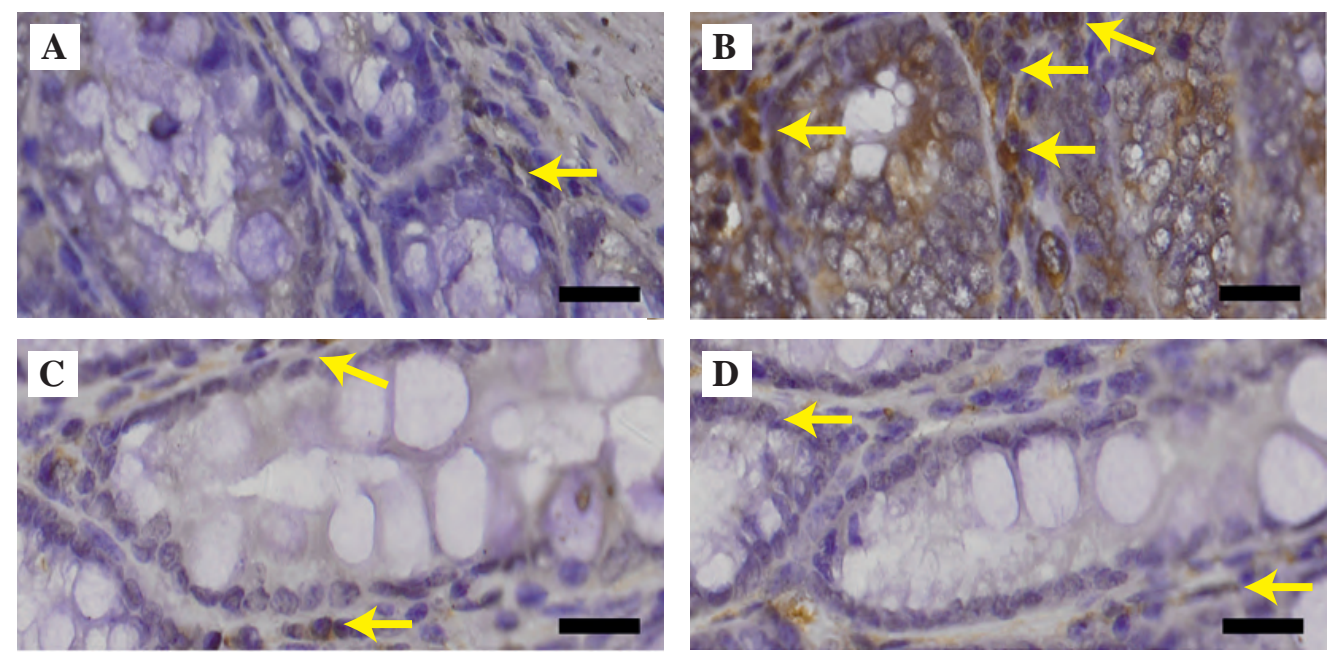

Figure 2. Results of immunohistochemistry staining to observe numbers of cells that express TNF- $\alpha$. Yellow arrow: TNF- $\alpha^{+}$cells (cells with a flat, brownish core). Black bar: $20 \mu \mathrm{m}$.

Table 2. Multiple comparisons of TNF- $\alpha$ expression.

\begin{tabular}{|c|c|c|c|c|c|}
\hline \multirow{2}{*}{ Groups } & \multicolumn{4}{|c|}{ Mann Whitney } & \multirow{2}{*}{$p$-value } \\
\hline & Sham & $\mathbf{U C}$ & $\mathrm{UC}+\mathrm{VD}$ & VD+UC & \\
\hline $\operatorname{Sham}(27.33 \pm 3.42)$ & - & $0.002 *$ & $0.002^{*}$ & $0.002 *$ & 0.000 \\
\hline UC (44.66 \pm 4.87$)$ & $0.002 *$ & - & $0.002 *$ & $0.041 *$ & \\
\hline $\mathrm{UC}+\mathrm{VD}(36.33 \pm 1.86)$ & $0.002 *$ & $0.002 *$ & - & 0.589 & \\
\hline VD+UC (38.68 \pm 3.68$)$ & $0.002 *$ & $0.002 *$ & 0.589 & - & \\
\hline
\end{tabular}

"Significancy was tested with Kruskal Wallis $(p<0.05)$. 

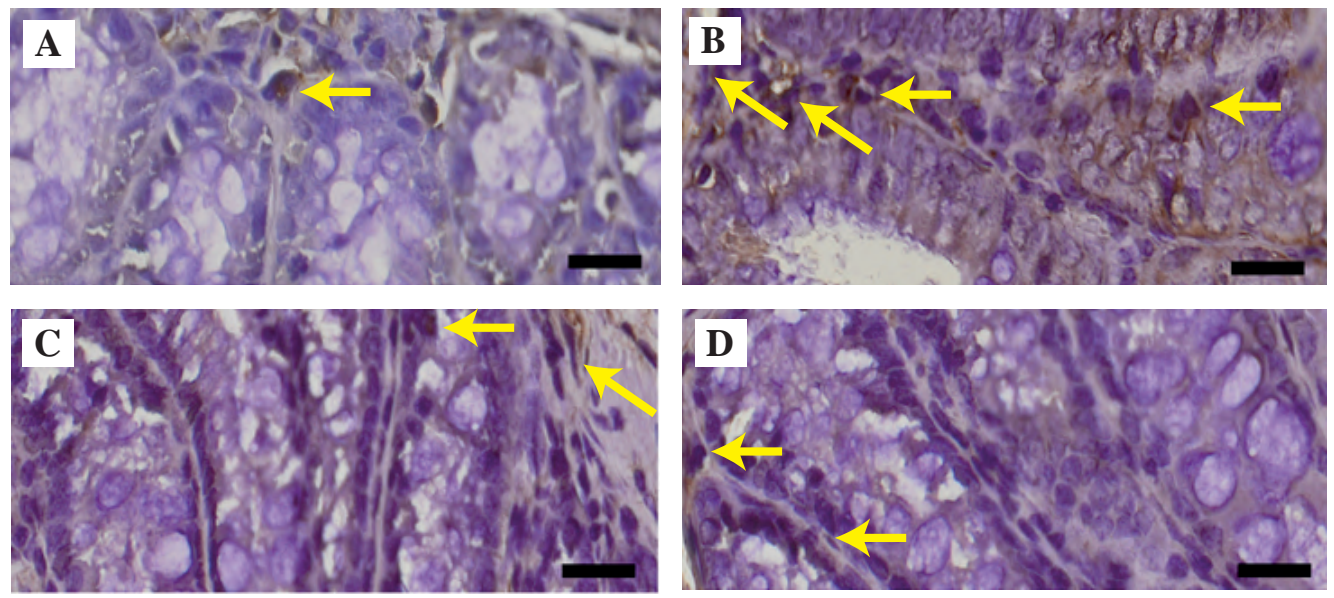

Figure 3. Results of immunohistochemistry staining to observed number of cells that express IL-6. Yellow arrow: IL-6 ${ }^{+}$cells (cells with a flat, brownish core). Black bar: $20 \mu \mathrm{m}$.

\section{Discussion}

At present, various treatments do not meet the full remission of the ulcerative condition and cause complications like anemia. Anemia is frequently found in patients at the time of diagnosis and correlates with the severity of the disease. $(16,17)$ Anemia in colitis can occur as a consequence of impaired absorption of iron, continuous blood loss in the acute phase, or even both.(18) As shown in this study, we found significant differences in hemoglobin levels among colitis mice compared to the Sham group. Hemoglobin levels increased with vitamin D3 administration, which suggested that vitamin D3 played a role in preventing or improving anemia as a complication of colitis.

Vitamin D3 is known as an immunomodulation agent in homeostasis and inflammation disease. Vitamin D3 reduced the expression of TNF- $\alpha$ and IL-6 colon in this study. As previously known, vitamin D3 can decrease the activation of NF- $\mathrm{kB}$ in a vitamin D receptor (VDR)- dependent manner. Vitamin D also blocks the TNF- $\alpha$ induced NF-KB activation. The deletion of VDR also ameliorates the regulation of inflammation by reducing I $\mathrm{I} B \alpha$ production. I $\kappa \mathrm{B} \alpha$ inhibits the NF- $\mathrm{KB}$ activity by preventing its translocation into the nucleus. $(19,20)$ Then, the levels of IL-6 may affect hemoglobin levels by increasing hepcidin production. Increased hepcidin production is inversely correlated with circulating iron levels. It binds ferroportin, the iron exporter, to prevent the release of iron from enterocyte into circulation.(18) This can be one of the underlying mechanisms of anemia in colitis because IL-6 production increases significantly and may lead to iron deficiency.

Previous studies have shown that vitamin D supplementation in clinical trials of UC patients significantly improved patients' quality of life, based on Inflammatory Bowel Disease Questionnaire-9 (IBDQ-9) and Simple Clinical Colitis Activity Index Questionnaire (SSCAIQ) questionnaires.(21) In this study, vitamin D3 administration improved the mucosal improvement, which was evaluated by MCHI score. This scoring system is a newly developed

Table 3. Multiple comparisons of IL-6 expression.

\begin{tabular}{|c|c|c|c|c|c|}
\hline \multirow{2}{*}{ Groups } & \multicolumn{4}{|c|}{ Mann Whitney } & \multirow{2}{*}{$p$-value } \\
\hline & Sham & UC & $\mathrm{UC}+\mathrm{VD}$ & $\mathrm{VD}+\mathrm{UC}$ & \\
\hline Sham (18.05 \pm 2.96$)$ & - & $0.002 *$ & $0.002 *$ & $0.009 *$ & 0.000 \\
\hline UC $(38.85 \pm 3.51)$ & $0.002 *$ & - & $0.002 *$ & $0.002 *$ & \\
\hline $\mathrm{UC}+\mathrm{VD}(24.78$ 0.79) & $0.002 *$ & $0.002 *$ & - & 0.937 & \\
\hline VD+UC (25.09 \pm 2.79$)$ & $0.009 *$ & $0.002 *$ & 0.937 & - & \\
\hline
\end{tabular}

\#Significancy was tested with Kruskal Wallis $(p<0.05)$. 
tool to evaluate mucosal damage and is claimed to be as reliable as endoscopic evaluation in pre-clinical studies.(15) The finding of increased mucosal improvement in this study indicated the role of vitamin D3 in preventing or treating anemia in colitis, as it reduced the possibility of continuous blood loss. Blood loss itself was included in the calculation of Mayo score to determine the severity of the disease with other endoscopic and clinical scales used.(22)

This study would reveal that there are other benefits of vitamin D3 administration to anemia, as one of the most frequent complications of ulcerative colitis. However, further research is required to understand the mechanism of vitamin D3 in the regulation of hepcidin production to increase hemoglobin levels. Further evaluation is needed to determine vitamin D3 levels in circulation, its toxicity, and other aspects to determine the most beneficial pathway as a therapeutic or preventive target in the development and severity of ulcerative colitis.

\section{Conclusion}

Before and after the colitis induction, vitamin D3 administration significantly reduced the expression of pro-inflammatory cytokines, especially TNF- $\alpha$ and IL-6. This treatment also improved the mucosal repair process, as showed in the histology studies and observed with the MCHI score. Moreover, vitamin D3 administration in this study improved anemia condition in UC, which was proved by significantly higher hemoglobin levels in the treatment groups. However, all these benefits of vitamin D3 still require further research to determine its therapeutic window and toxicity.

\section{References}

1. Randhawa PK, Singh K, Singh N, Jaggi AS. A review on chemicalinduced inflammatory bowel disease models in rodents. Korean $\mathrm{J}$ Physiol Pharmacol. 2014; 18: 279-88.

2. Mustika S, Triana N. The prevalence, profile, and risk factor of patients with ulcerative colitis at Dr. Saiful Anwar Malang General Hospital. Indones J Gastroenterol Hepatol Dig Endosc. 2016; 17: $16-20$.

3. Basheer W, Kunde D, Eri R. Role of chemokine ligand CCL20 and its receptor CCR6 in intestinal inflammation. Immunol Infect Dis. 2013; 1: 30-7.

4. Antunes CV de A, Hallack Neto AE, Nascimento CR de A, Chebli LA, Moutinho ILD, Pinheiro B do V, et al. Anemia in inflammatory bowel disease outpatients: prevalence, risk factors, and etiology. Biomed Res Int. 2015; 2015: 728925. doi: $10.1155 / 2015 / 728925$.

5. Alves RA, Miszputen SJ, Figueiredo MS. Anemia in inflammatory bowel disease: prevalence, differential diagnosis and association with clinical and laboratory variables. Sao Paulo Med J. 2014; 132: 140-6.

6. Kaitha S, Bashir M, Ali T. Iron deficiency anemia in inflammatory bowel disease. World J Gastrointest Pathophysiol. 2015; 6: 62-72.

7. Ananthakrishnan AN, Khalili H, Konijeti GG, Higuchi LM, De Silva P, Korzenik JR, et al. A prospective study of long-term intake of dietary fiber and risk of Crohn's disease and ulcerative colitis. Gastroenterology. 2013; 145: 970-7.

8. Abraham BP, Prasad P, Malaty HM. Vitamin D deficiency and corticosteroid use are risk factors for low bone mineral density in inflammatory bowel disease patients. Dig Dis Sci. 2014; 59: 187884.

9. AlSheikh MH, Almubayadh SI. Effect of vitamin D supplementation on insulin, fasting blood glucose, and waist-hip ratio in young females with pre-existing vitamin D deficiency. Indones Biomed J. 2019; 11: 42-7.

10. Lai YH, Fang TC. The pleiotropic effect of vitamin D. ISRN Nephrol. 2013; 2013: 898125. doi: 10.5402/2013/898125.

11. Dai Z, Tan B, Yang H, Wang O, Qian JJ, Lv H. 1,25-hydroxyvitamin D relieves colitis in rats via downregulation of toll-like receptor 9 expression. Croat Med J. 2015; 56: 515-24.

12. Dionne S, Calderon MR, White JH, Memari B, Elimrani I, Adelson $\mathrm{B}$, et al. Differential effect of vitamin D on NOD2-and TLR-induced cytokines in Crohn's disease. Mucosal Immunol. 2014; 7: 1405-15.

13. Reich KM, Fedorak RN, Madsen K, Kroeker KI. Vitamin D improves inflammatory bowel disease outcomes: basic science and clinical review. World J Gastroenterol WJG. 2014; 20: 4934-47.

14. Zughaier SM, Alvarez JA, Sloan JH, Konrad RJ, Tangpricha V. The role of vitamin $\mathrm{D}$ in regulating the iron-hepcidin-ferroportin axis in monocytes. J Clin Transl Endocrinol. 2014; 1(1): e19-e25.

15. Koelink PJ, Wildenberg ME, Stitt LW, Feagan BG, Koldijk M, van't Wout $\mathrm{AB}$, et al. Development of reliable, valid and responsive scoring systems for endoscopy and histology in animal models for inflammatory bowel disease. J Crohn's Colitis. 2018; 12: 794-803.

16. Tulewicz-Marti E, Moniuszko A, Rydzewska G. Management of anemia in inflammatory bowel disease: a challenge in everyday clinical practice. Prz Gastroenterol. 2017; 12: 239-43.

17. Baumgart D, Baumgart DC. Crohn's disease and ulcerative colitis. Switzerland: Springer; 2017.

18. Rogler G, Vavricka S. Anemia in inflammatory bowel disease: an under-estimated problem? Front Med. 2015; 1: 58. doi: 10.3389/ fmed.2014.00058.

19. Wu-Wong JR. Potential for vitamin D receptor agonists in the treatment of cardiovascular disease. Br J Pharmacol. 2009; 158: 395-412.

20. Chen Y, Zhang J, Ge X, Du J, Deb DK, Li YC. Vitamin D receptor inhibits nuclear factor $\mathrm{\kappa B}$ activation by interacting with IкB kinase $\beta$ protein. J Biol Chem. 2013; 288: 19450-8.

21. Karimi S, Tabataba-vakili S, Yari Z, Alborzi F, Hedayati M, Ebrahimi-Daryani $\mathrm{N}$, et al. The effects of two vitamin D regimens on ulcerative colitis activity index, quality of life and oxidant/ anti-oxidant status. Nutr J. 2019;18: 16. doi: 10.1186/s12937-0190441-7.

22. Schroeder KW, Tremaine WJ, Ilstrup DM. Coated oral 5 -aminosalicylic acid therapy for mildly to moderately active ulcerative colitis. N Engl J Med. 1987; 317: 1625-9. 